\title{
Assistência de enfermagem na orientação e cuidados de pacientes renais crônicos submetidos a diálise peritoneal: uma revisão integrativa
}

\author{
Wady Wendler Soares Veras e Silva \\ Graduando do curso de enfermagem da Universidade Estadual do Piauí (UESPI) \\ $\triangle$ wadywendler12@gmail.com \\ Maria Izabel Félix Rocha \\ Graduanda do curso de enfermagem da Universidade Estadual do Piauí (UESPI) \\ Kelly Sivocy Sampaio Teixeira \\ Professora Doutora do curso bacharelado de enfermagem da Universidade Estadual do Piauí (UESPI) \\ Francisco Artur e Silva Filho \\ Professor Doutor do curso bacharelado de enfermagem da Universidade Estadual do Piauí (UESPI)
}

Recebido em 3 de maio de 2021

Aceito em 8 de setembro de 2021

\section{Resumo:}

O papel do enfermeiro na unidade de diálise peritoneal (DP) é extremamente relevante, pois ele planeja os cuidados, orientações e soluciona problemas que interfiram no método dialítico. O objetivo desse estudo é descrever o papel do enfermeiro na prestação da assistência em pacientes renais crônicos submetidos a DP. Esse estudo apresenta pesquisas nas bases de dados PubMed, LILACS e SciELo, no período de 2009 a 2019. Com manuscritos no idioma português, inglês e espanhol, os descritores utilizados foram "Diálise peritoneal"; "Cuidados de Enfermagem"; "Insuficiência Renal crônica" e "Peritoneal Dialysis"; "Nursing Care"; "Renal Insufficiency, Chronic". Depois das buscas nas bases de dados selecionadas, foram escolhidos 147 artigos. Ademais, foi realizada uma análise que resultou na amostra de 10 documentos. Após a leitura completa dos artigos selecionados, foram elaboradas quatro categorias de análise, que são: "A DP como escolha do paciente por modalidade de tratamento"; "Orientações necessárias que o enfermeiro deve fornecer para os pacientes em DP"; "Principais diagnósticos de enfermagem e intervenções que o enfermeiro pode realizar em pacientes com DP"; "A assistência prestada pelo enfermeiro ao paciente em processo de DP". Observou-se que a maioria dos estudos destaca a importância do enfermeiro no auxilio das orientações e cuidados ao paciente dialítico, como a higienização das mãos, dos materiais utilizados, cuidados no domicilio e do local da inserção do cateter. Por fim, o enfermeiro é imprescindível no tratamento da DP, pois o mesmo facilita e traz uma maior eficácia ao processo, melhorando a qualidade de vida e diminuindo possíveis complicações.

Palavras-chave: Diálise peritoneal, Cuidados de Enfermagem, Insuficiência Renal crônica, Educação em Saúde.

\section{Nursing assistance in the guidance and care of chronic renal patients submitted to peritoneal dialysis: an integrative review}

\begin{abstract}
:
The role of the nurse in the peritoneal dialysis unit is extremely relevant, as they plan care, guidance and solve problems that interfere with the dialysis method. The objective of this study to describe the role of nurses in providing guidance and care for chronic renal patients submitted to DP. This
\end{abstract}


integrative review of the literature consists of searches in the databases PubMed, LILACS e SciELo, in the period from 2009 to 2019. With manuscripts in Portuguese, English and Spanish, the descriptors used were "Diálise peritoneal"; "Cuidados de Enfermagem"; "Insuficiência Renal crônica" e "Peritoneal Dialysis"; "Nursing Care"; "Renal Insufficiency, Chronic". After searching the selected databases, 147 articles were chosen. Then, an analysis was performed that resulted in a sample of 10 documents. After a complete reading of the selected articles, four categories of analysis were elaborated, which are they: "PD as patient choice by treatment modality"; "Necessary guidelines that nurses should provide for patients on PD"; "Main nursing diagnoses and interventions that nurses can perform in patients with PD"; "The assistance provided by the nurse to the patient in the PD process". It was observed that most studies highlight the importance of nurses in helping to provide guidelines and care for dialysis patients, such as hand hygiene, materials used, care at home and the location of catheter insertion. Finally, the nurse is essential in the treatment of PD, as it facilitates and brings greater effectiveness to the process, improving the quality of life and reducing possible complications. Keywords: Peritoneal Dialysis, Nursing Care, Renal Insufficiency, Chronic, Health Education.

\section{Asistencia de enfermería en la orientación y atención de pacientes renales crónicos sometidos a diálisis peritoneal: una revisión integradora}

\section{Resumen:}

El papel de la enfermera en la unidad de diálisis peritoneal es extremadamente relevante ya que planifican la atención, orientan y resuelven problemas que interfieren con el método de diálisis. El objetivo de este estudio es describir el papel del enfermero en la orientación y atención de los pacientes renales crónicos sometidos a diálisis peritoneal. Esta revisión integradora de la literatura consiste en búsquedas en las bases de datos PubMed, LILACS e SciELo, en el período de 2009 a 2019. Con manuscritos en portugués, inglés y español, los descriptores utilizados fueron "Diálise peritoneal"; "Cuidados de Enfermagem"; "Insuficiência Renal crônica" e "Diálisis peritoneal"; "Cuidado de enfermera"; "Insuficiencia renal crónica". Después de buscar en las bases de datos seleccionadas, se eligieron 147 artículos. Luego, se realizó un análisis que resultó en una muestra de 10 documentos. Luego de una lectura completa de los artículos seleccionados, se elaboraron cuatro categorías de análisis, las cuales son: "DP como elección del paciente por modalidad de tratamiento"; "Pautas necesarias que las enfermeras deben brindar a los pacientes en DP"; "Principales diagnósticos e intervenciones de enfermería que las enfermeras pueden realizar en pacientes con DP"; "La asistencia brindada por la enfermera al paciente en el proceso de DP". Se observó que la mayoría de los estudios destacan la importancia de las enfermeras para ayudar a brindar pautas y cuidados a los pacientes en diálisis, como la higiene de las manos, los materiales utilizados, los cuidados en el hogar y el lugar de inserción del catéter. Finalmente, la enfermera es fundamental en el tratamiento de la DP, ya que facilita y aporta mayor efectividad al proceso, mejorando la calidad de vida y reduciendo posibles complicaciones.

Palabras clave: Diálisis Peritoneal, Atención de Enfermería, Insuficiencia Renal Crónica, Educación en Salud.

\section{INTRODUÇÃO}

A Doença Renal Crônica (DRC) é caracterizada por alterações heterogêneas que afetam a função renal, onde suas principais causas são a hipertensão arterial, doenças autoimunes, diabetes e doenças genéticas (BRASIL, 2014). De acordo com a Sociedade Brasileira de Nefrologia (SBN), nos últimos 12 anos houve um aumento de $4 \%$ nos casos de DRC por diabetes 
(NEVES et al, 2020). Além disso, ocorre um agravamento progressivo e irreversível, onde há dificuldade do corpo em manter a homeostasia do organismo. Logo, haverá necessidade de tratamento para substituir a função dos rins (MUSCAT et al., 2021).

Um dos possíveis tratamentos para essa patologia é a Diálise Peritoneal (DP), foco desse estudo. Essa terapia é realizada através da inserção de 1 a 3 litros de uma solução salina contendo dextrose (solução de diálise) na cavidade peritoneal. Por meio de uma difusão e de ultrafiltração, os matérias tóxicos para o nosso organismo e o excesso de água se movem do nosso corpo para a solução de diálise (DA SILVA MORAES et al., 2018). Segundo ainda a SBN, em julho de 2018, o número total estimado de pacientes em diálise foi de 133.464 (NEVES et al., 2020).

Essa modalidade de tratamento pode ser realizada no ambiente domiciliar, no qual acaba se tornado uma alternativa terapêutica que traz uma liberdade para o paciente, uma maior qualidade de vida e consequentemente uma melhora no seu quadro clínico. No entanto, esse procedimento requer responsabilidade e conhecimento de quem está o executando. A decisão do cliente pelo método dialítico deve ser uma escolha conjunta entre ele e a equipe de nefrologia, respeitando as suas necessidades (BEZERRA, 2017; MENDES et al., 2017).

Em síntese, sabe-se que o enfermeiro tem sua importância na unidade de Diálise Peritoneal. É ele quem planeja os cuidados e orientações que contribuem para a diminuição de problemas que possam interferir no método dialítico A adesão ao tratamento por DP em ambiente domiciliar pode ser feita por um profissional de enfermagem, ensinando os procedimentos de forma segura e prática, diminuindo o risco de infecções na região peritoneal, tendo uma maior atenção e cuidado com o cateter (DA SILVA, 2019). Portanto, o presente estudo tem o objetivo de descrever o papel do enfermeiro na prestação de orientações e cuidados em pacientes renais crônicos submetidos a diálise peritoneal.

\section{METODOLOGIA}

Trata-se de uma revisão integrativa da literatura, em que se agrupou diversos resultados de pesquisas obtidos em artigos de base de dados online, com o objetivo de obter 
uma ampla compreensão do fenômeno estudado. Esse estudo considerou as 6 partes do processo da elaboração de uma revisão integrativa: 1) Elaboração da pergunta norteadora, 2) Busca ou amostragem na literatura, 3) Coleta de dados, 4) Análise crítica dos estudos incluídos, 5) Discussão dos resultados e 6) Apresentação da revisão integrativa (DE SOUSA et al., 2017).

Para que os objetivos fossem respondidos, foi elaborada a seguinte questão norteadora: Quais as orientações e cuidados fornecidos pelos enfermeiros para os pacientes submetidos a diálise peritoneal?

Para a busca dos descritores utilizados nessa pesquisa, foi consultado o DeCS (Descritores em Ciências de Saúde) e o MeSH (Medical Subjects Headings). As palavras utilizadas no DeCS foram "Diálise peritoneal"; "Cuidados de Enfermagem"; "Insuficiência Renal crônica"; Como MeSH, foram usados "Peritoneal Dialysis"; "Nursing Care"; "Renal Insufficiency, Chronic". Além disto, as bases de dados utilizadas foram a US National Library of Medicine, Literatura Latino-Americana e do Caribe em Ciências da Saúde a Scientific Eletronic Library Online (PubMed, LILACS e SciELo). Outrossim, combinações dos seguintes descritores e operadores booleanos foram utilizadas para se ter os manuscritos na integra: Diálise peritoneal and Cuidados de Enfermagem and Insuficiência Renal crônica; Peritoneal Dialysis and Nursing Care and Renal Insufficiency, Chronic.

Além disto os critérios de inclusão utilizados para o desenvolvimento desse estudo foram: manuscritos no idioma português, inglês e espanhol disponibilizados nas bases de dados e publicados entre os anos de 2009 e 2019, com o objetivo de obter artigos relacionados a temática escolhida e que fossem recentes. Os critérios de exclusão foram: documentos que não se enquadravam nos critérios de inclusão, bem como estudos de casos, revisões de literaturas e artigos que não respondiam aos objetivos e a pergunta norteadora propostos para esse estudo.

A seleção dos artigos para análise foi realizada primeiramente pela leitura dos títulos. Aqueles que não atenderam ao objetivo da pesquisa foram excluídos. Dos que ficaram, foi lido o resumo de todos os estudos encontrados na busca em cada uma das bases de dados. Foram descartados os artigos cujos resumos não entraram em consonância com a proposta desta revisão. O passo seguinte foi selecionar os artigos na íntegra e analisá-los. 
Os manuscritos selecionados após sua leitura por completo, foram tratados por meio do fichamento de ursi, um instrumento que permite avaliar cada artigo individualmente, resumindo tanto sua metodologia, quanto seus resultados, o que possibilitou uma melhor organização dos dados, facilitando a análise dos estudos posteriormente. Os tópicos de interesse foram: o título do artigo, ano e país de publicação, delineamento do tipo de estudo, idioma, autores, ano da publicação, amostra final e desfecho. A figura 1 mostra o fluxograma do processo de seleção dos manuscritos para análise.

Figura 1 - Fluxograma explicativo do processo de seleção dos manuscritos.

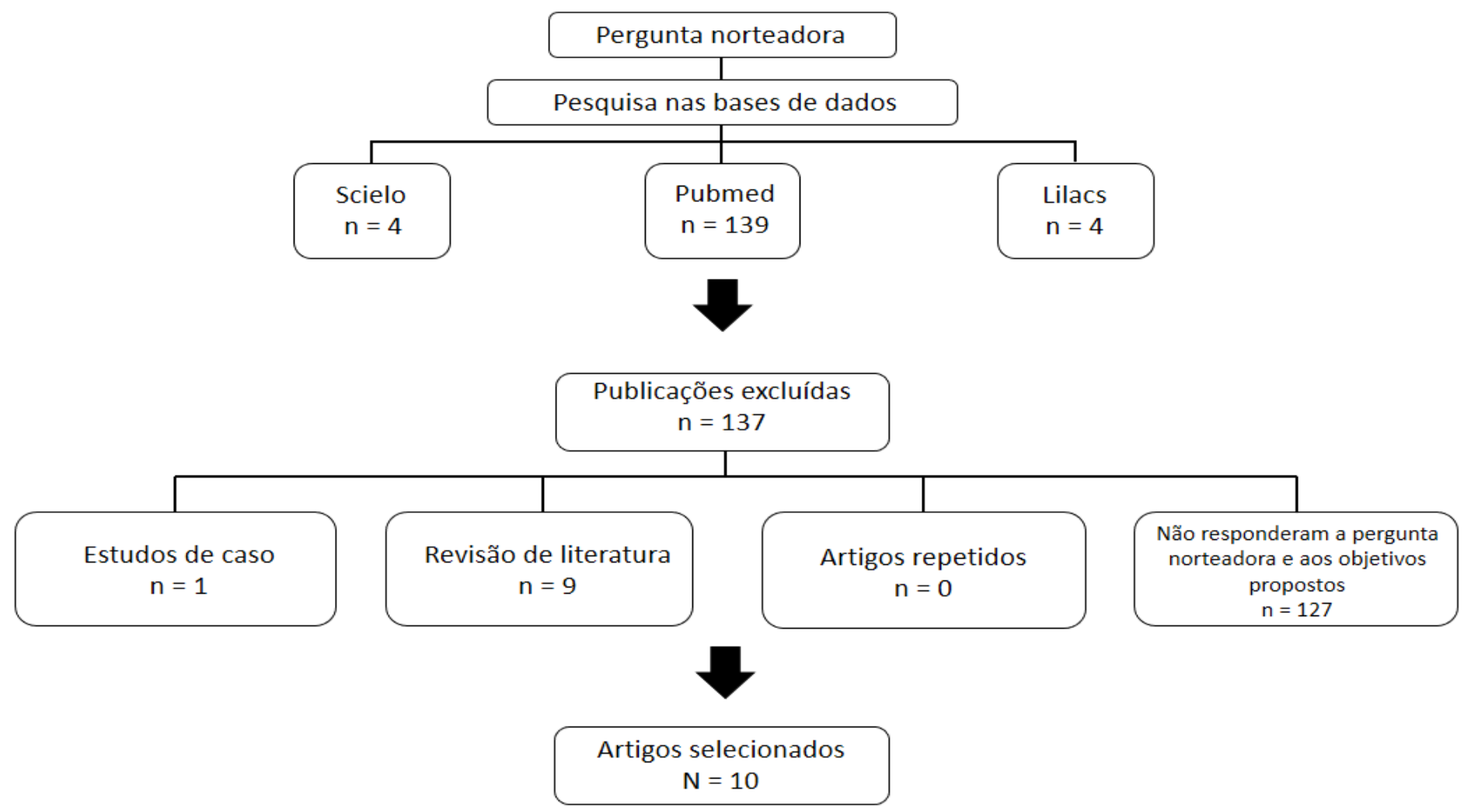

Fonte: Autoria Própria.

\section{RESULTADOS}

A busca nas bases de dados selecionadas resultou em 147 artigos. Depois da leitura minuciosa seguindo de título, resumo e artigo completo, sempre respeitando os critérios de inclusão e exclusão foram eleitos 10 artigos que compõem a amostra final. Desta amostra 
final, sete foram obtidos na base de dados PubMed, dois na base de dados da Scielo, enquanto um foi encontrado na base de dados do Lilacs.

Dos artigos utilizados na revisão, 3 (30\%) artigos foram publicados em 2019, 1 (10\%) artigo foi publicado em 2018, 1 (10\%) artigo foi publicado em 2017, 2 (20\%) artigos foram publicados em 2016, 2 (20\%) artigos foram publicados em 2012 e 1 (10\%) foi publicado em 2010. Houve uma diversificação nos locais de publicação, no qual 3 (30\%) foram publicados no Brasil, 2 (20\%) foram publicados no Taiwan, 2 (20\%) foram publicados no China, 1 (10\%) foi publicado na Turquia, 1 (10\%) foi publicado na Inglaterra e 1 (10\%) publicado no Japão. 0 Quadro 1 apresenta a síntese de cada um dos artigos selecionados.

Quadro 1 - Caracterização dos estudos incluídos na revisão integrativa acerca do tema Assistência de enfermagem na orientação e cuidados de pacientes renais crônicos submetidos a diálise peritoneal segundo os autores/ano, título do manuscrito, Revista, local do estudo, Metodologia e principais resultados/ conclusões.

\begin{tabular}{|c|c|c|c|c|c|}
\hline $\begin{array}{l}\text { AUTOR/ } \\
\text { ANO }\end{array}$ & TÍTULO & REVISTA & LOCAL & METODOLOGIA & $\begin{array}{l}\text { RESULTADOS E } \\
\text { CONCLUSÕES }\end{array}$ \\
\hline $\begin{array}{l}\text { Campos, } \\
\text { M. X. B. et } \\
\text { al. } 2019\end{array}$ & $\begin{array}{l}\text { Pacientes } \\
\text { em diálise } \\
\text { peritoneal } \\
: \\
\text { associação } \\
\text { entre } \\
\text { diagnóstic } \\
\text { os de } \\
\text { enfermag } \\
\text { em e seus } \\
\text { componen } \\
\text { tes. }\end{array}$ & $\begin{array}{l}\text { Acta Paulista } \\
\text { de } \\
\text { Enfermagem }\end{array}$ & Brasil & $\begin{array}{l}\text { Estudo de validação de } \\
\text { conteúdo realizado com } \\
\text { seis enfermeiros } \\
\text { nefrologistas, os quais } \\
\text { participaram no presente } \\
\text { estudo como peritos. } \\
\text { Utilizou-se a técnica de } \\
\text { grupo focal. Para análise } \\
\text { dos dados foi utilizada a } \\
\text { regressão logística. }\end{array}$ & 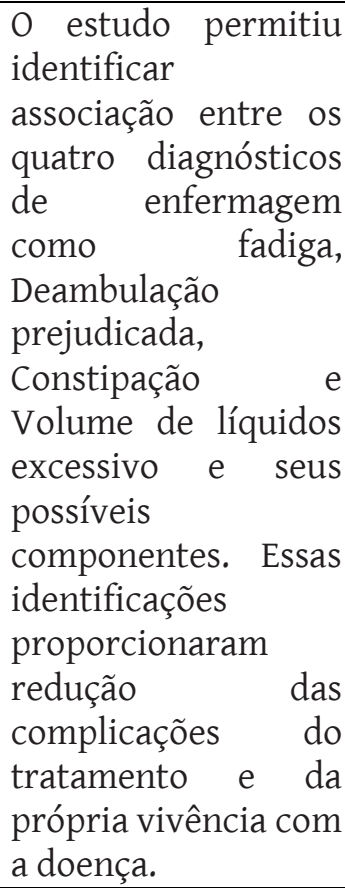 \\
\hline $\begin{array}{l}\text { Chen, H. L.; } \\
\text { Tarng, D. } \\
\text { C.; Huang, } \\
\text { L. H. et al. } \\
2019\end{array}$ & $\begin{array}{l}\text { Risk } \\
\text { factors } \\
\text { associated } \\
\text { with } \\
\text { outcomes } \\
\text { of } \\
\text { peritoneal } \\
\text { dialysis in }\end{array}$ & Medicine & Taiwan & $\begin{array}{l}\text { O estudo inscreveu } \\
\text { pacientes do ESRD que } \\
\text { receberam cateteres de DP } \\
\text { em um hospital terciário no } \\
\text { norte de Taiwan. Utilizando } \\
\text { um modelo de regressão de } \\
\text { riscos concorrentes, } \\
\text { revisamos os dados clínicos }\end{array}$ & $\begin{array}{lr}\text { No estudo, foram } \\
\text { inscritos } & 514 \\
\text { pacientes com } & \text { DP } \\
\text { entre } 2001 & \text { e } \\
\text { 2013. Foi descoberto } \\
\text { que, o gênero } \\
\text { masculino } r \text { está } \\
\text { associado a mais }\end{array}$ \\
\hline
\end{tabular}




\begin{tabular}{|c|c|c|c|c|c|}
\hline & $\begin{array}{l}\text { Taiwan: } \\
\text { An } \\
\text { analysis } \\
\text { using a } \\
\text { competin } \\
\text { g risk } \\
\text { model. }\end{array}$ & & & $\begin{array}{l}\text { e os analisamos em termos } \\
\text { de tempo para falha técnica } \\
\text { e desfechos clínicos, } \\
\text { incluindo peritonite e } \\
\text { mortalidade relacionadas à } \\
\text { DP. }\end{array}$ & $\begin{array}{l}\text { falhas técnicas de } \\
\text { cateteres em DP do } \\
\text { que em mulheres. } \\
\text { Diabetes mellitus foi } \\
\text { o único fator de risco } \\
\text { para peritonite } \\
\text { relacionada à DP. }\end{array}$ \\
\hline $\begin{array}{l}\text { Karadag, E. } \\
2019\end{array}$ & $\begin{array}{l}\text { The effect } \\
\text { of a self- } \\
\text { managem } \\
\text { ent } \\
\text { program } \\
\text { on hand- } \\
\text { washing/ } \\
\text { mask- } \\
\text { wearing } \\
\text { behaviour } \\
\text { s and self- } \\
\text { efficacy } \\
\text { level in } \\
\text { peritoneal } \\
\text { dialysis } \\
\text { patients: a } \\
\text { pilot } \\
\text { study. }\end{array}$ & $\begin{array}{l}\text { Journal of } \\
\text { renal care }\end{array}$ & Turquia & $\begin{array}{l}\text { A amostra do estudo } \\
\text { consistiu em } 30 \text { pacientes } \\
\text { que estavam em diálise } \\
\text { peritoneal. Os pacientes } \\
\text { receberam um questionário } \\
\text { sobre Características } \\
\text { Sociodemográficas e de } \\
\text { Doenças e a Escala de } \\
\text { Autoeficácia. Após } 6 \text { meses, } \\
\text { essa escolada foi aplicada } \\
\text { novamente avaliando } \\
\text { perguntas sobre } \\
\text { comportamentos de } \\
\text { lavagem de mãos e uso de } \\
\text { máscaras. }\end{array}$ & $\begin{array}{l}\text { Neste estudo, } \\
\text { verificou-se que o } \\
\text { programa de } \\
\text { autogestão baseado } \\
\text { na teoria de } \\
\text { autoeficácia de } \\
\text { Bandura causou uma } \\
\text { mudança positiva } \\
\text { nos comportamentos } \\
\text { de lavagem das mãos } \\
\text { / uso de máscaras } \\
\text { dos pacientes e que } \\
\text { melhorou o nível de } \\
\text { autoeficácia. }\end{array}$ \\
\hline $\begin{array}{l}\text { Zee, J. et al } \\
.2018\end{array}$ & $\begin{array}{l}\text { Perceptio } \\
\text { ns about } \\
\text { the } \\
\text { dialysis } \\
\text { modality } \\
\text { decision } \\
\text { process } \\
\text { among } \\
\text { peritoneal } \\
\text { dialysis } \\
\text { and in- } \\
\text { center } \\
\text { hemodialy } \\
\text { sis } \\
\text { patients. }\end{array}$ & $\begin{array}{l}\text { BMC } \\
\text { nephrology }\end{array}$ & Inglaterra & $\begin{array}{l}\text { Uma pesquisa de } 39 \\
\text { perguntas foi desenvolvida } \\
\text { em colaboração com um } \\
\text { painel consultivo multi- } \\
\text { stakeholder para avaliar a } \\
\text { percepção dos pacientes } \\
\text { sobre DP ou hemodiálise no } \\
\text { centro (HD). As respostas } \\
\text { da pesquisa foram } \\
\text { comparadas entre } \\
\text { pacientes com DP e HD no } \\
\text { centro utilizando } \\
\text { estatísticas descritivas, } \\
\text { modelos de equação de } \\
\text { estimativas logísticas } \\
\text { ajustadas e modelos de } \\
\text { regressão mista linear. }\end{array}$ & \begin{tabular}{lr}
\multicolumn{2}{l}{ Os participantes da } \\
DP estavam mais \\
envolvidos r no \\
processo de decisão \\
da modalidade em \\
comparação com os \\
participantes no \\
centro de HD. Para \\
ambas & as \\
modalidades, há \\
espaço r para \\
melhorias na \\
educação do paciente \\
e outro suporte para \\
os pacientes que \\
escolhem r uma \\
modalidade re de \\
diálise.
\end{tabular} \\
\hline $\begin{array}{l}\text { Abud, A. C. } \\
\text { F, et al. } \\
2017\end{array}$ & $\begin{array}{l}\text { Ambiênci } \\
\text { a } \\
\text { domiciliar } \\
\text { para a } \\
\text { realização } \\
\text { da diálise } \\
\text { peritoneal }\end{array}$ & $\begin{array}{l}\text { Revista } \\
\text { Enfermagem } \\
\text { UERJ }\end{array}$ & Brasil & $\begin{array}{l}\text { Estudo quantitativo, } \\
\text { transversal, realizado com } \\
90 \text { pacientes em diálise } \\
\text { peritoneal. Os dados foram } \\
\text { obtidos por meio de } \\
\text { entrevista e observação } \\
\text { direta no domicílio. }\end{array}$ & $\begin{array}{l}\text { Observou-se que a DP } \\
\text { era realizada } \\
\text { principalmente no } \\
\text { quarto. A maioria das } \\
\text { casas possuía pias } \\
\text { para higiene das } \\
\text { mãos, água tratada, } \\
\text { armazenamento }\end{array}$ \\
\hline
\end{tabular}




\begin{tabular}{|c|c|c|c|c|c|}
\hline & & & & & $\begin{array}{l}\text { adequado de } \\
\text { material e esgoto, } \\
\text { onde os resíduos } \\
\text { eram descartados. } \\
\text { No entanto, os outros } \\
\text { pacientes que não } \\
\text { apresentavam esses } \\
\text { meios estavam se } \\
\text { expondo a riscos que } \\
\text { podem agravar seu } \\
\text { estado de saúde. }\end{array}$ \\
\hline $\begin{array}{l}\text { Chiang, P. } \\
\text { C. et al. } \\
2016\end{array}$ & $\begin{array}{l}\text { Factors } \\
\text { Associate } \\
d \text { with the } \\
\text { Choice of } \\
\text { Peritoneal } \\
\text { Dialysis in } \\
\text { Patients } \\
\text { with End- } \\
\text { Stage } \\
\text { Renal } \\
\text { Disease. }\end{array}$ & $\begin{array}{l}\text { BioMed } \\
\text { research } \\
\text { international }\end{array}$ & Taiwan & $\begin{array}{l}\text { Nesse estudo foi revisado } \\
\text { retrospectivamente as } \\
\text { características } \\
\text { demográficas, as cargas de } \\
\text { doenças pessoais e as } \\
\text { modalidades finais de } \\
\text { diálise de todos os } \\
\text { pacientes que receberam o } \\
\text { programa multidisciplinar } \\
\text { de educação em pre-diálise } \\
\text { (MPE) e iniciaram sua } \\
\text { primeira terapia de diálise } \\
\text { no hospital de estudos } \\
\text { entre } 1^{\circ} \text { de janeiro de } 2008 \text { e } \\
30 \text { de junho de } 2013 \text {. }\end{array}$ & $\begin{array}{l}\text { Este estudo indica } \\
\text { que as características } \\
\text { dos pacientes que } \\
\text { mais influenciam no } \\
\text { tratamento da diálise } \\
\text { peritoneal são a } \\
\text { idade, o nível de } \\
\text { escolaridade e nível } \\
\text { de atividades da vida } \\
\text { diária (AVD). }\end{array}$ \\
\hline $\begin{array}{l}\text { Silva, R. A. } \\
\text { R. et al. } \\
2016\end{array}$ & $\begin{array}{l}\text { Diagnóstic } \\
\text { os, } \\
\text { resultados } \\
\text { e } \\
\text { intervenç } \\
\text { ões de } \\
\text { enfermag } \\
\text { em para } \\
\text { pacientes } \\
\text { em diálise } \\
\text { peritoneal }\end{array}$ & $\begin{array}{l}\text { Acta Paulista } \\
\text { de } \\
\text { Enfermagem }\end{array}$ & Brasil & $\begin{array}{l}\text { Estudo transversal com } 68 \\
\text { pacientes em centro de } \\
\text { referência para doenças } \\
\text { renais, seguindo as etapas: } \\
\text { elaboração dos } \\
\text { Diagnósticos de } \\
\text { Enfermagem a partir da } \\
\text { NANDA-Internacional; } \\
\text { proposta inicial de } \\
\text { resultados e intervenções } \\
\text { de enfermagem, conforme } \\
\text { a Nursing Outcomes } \\
\text { Classification e Nursing } \\
\text { Interventions } \\
\text { Classification; e elaboração } \\
\text { de um plano de cuidados } \\
\text { com validação por } \\
\text { especialistas. }\end{array}$ & $\begin{array}{l}\text { O estudo permitiu } \\
\text { identificar } \\
\text { diagnósticos e } \\
\text { selecionar resultados } \\
\text { e intervenções de } \\
\text { enfermagem para } \\
\text { aplicação na prática } \\
\text { clínica, com vistas a } \\
\text { subsidiar o processo } \\
\text { de cuidado e o } \\
\text { conhecimento das } \\
\text { taxonomias de } \\
\text { enfermagem. }\end{array}$ \\
\hline $\begin{array}{l}\text { Yang Z. et } \\
\text { al. } 2012\end{array}$ & $\begin{array}{l}\text { Advanced } \\
\text { nursing } \\
\text { experienc } \\
\mathrm{e} \quad \text { is } \\
\text { beneficial } \\
\text { for } \\
\text { lowering } \\
\text { the }\end{array}$ & $\begin{array}{l}\text { Peritoneal } \\
\text { dialysis } \\
\text { international }\end{array}$ & China & $\begin{array}{l}\text { A regressão de Cox } \\
\text { multivariada foi utilizada } \\
\text { para analisar a associação } \\
\text { de riscos para peritonite de } \\
\text { todas as causas e por } \\
\text { bactérias gram-positivas } \\
\text { com o treinamento de } \\
\text { pacientes fornecido por }\end{array}$ & $\begin{array}{l}\text { A experiência em } \\
\text { medicina geral de } \\
\text { enfermeiros pode } \\
\text { ajudar a diminuir o } \\
\text { risco de peritonite } \\
\text { por bactérias gram- } \\
\text { positivas entre } \\
\text { pacientes com DP. }\end{array}$ \\
\hline
\end{tabular}




\begin{tabular}{|c|c|c|c|c|c|}
\hline & $\begin{array}{l}\text { peritonitis } \\
\text { rate in } \\
\text { patients } \\
\text { on } \\
\text { peritoneal } \\
\text { dialysis. }\end{array}$ & & & $\begin{array}{l}\text { enfermeiros em diferentes } \\
\text { níveis de experiência. }\end{array}$ & $\begin{array}{l}\text { Esses dados são os } \\
\text { primeiros a indicar } \\
\text { que a experiência de } \\
\text { enfermagem em } \\
\text { outras áreas que não } \\
\text { a prática de DP pode } \\
\text { ser vital no } \\
\text { treinamento de } \\
\text { pacientes com DP. }\end{array}$ \\
\hline $\begin{array}{l}\text { Imada, A. } \\
2012\end{array}$ & $\begin{array}{l}\text { Care for } \\
\text { Patients } \\
\text { Carrying } \\
\text { Out } \\
\text { Dialysis } \\
\text { Therapy } \\
\text { at Home. }\end{array}$ & $\begin{array}{l}\text { Home } \\
\text { Dialysis in } \\
\text { Japan. }\end{array}$ & Japão & $\begin{array}{l}\text { Resumo de pontos } \\
\text { importantes } \\
\text { importância } \\
\text { enfermagem no auxílio a } \\
\text { diálise domiciliar para cada } \\
\text { tratamento, podendo ser } \\
\text { diálise } \\
\text { ambulatória peritoneal } \\
\text { (DPAC) e contínua } \\
\text { domiciliar (HDD) }\end{array}$ & $\begin{array}{l}\text { Nesse estudo mostra } \\
\text { que a taxa de } \\
\text { utilização de DPAC e } \\
\text { HDD em casa ainda é } \\
\text { baixo no Japão. }\end{array}$ \\
\hline $\begin{array}{l}\text { Chow, S. K. } \\
\text { W, Wong, } \\
\text { F. K. } 2010\end{array}$ & $\begin{array}{l}\text { Health- } \\
\text { related } \\
\text { quality of } \\
\text { life in } \\
\text { patients } \\
\text { undergoin } \\
\text { g } \\
\text { peritoneal } \\
\text { dialysis: } \\
\text { effects of a } \\
\text { nurse-led } \\
\text { case } \\
\text { managem } \\
\text { ent } \\
\text { program } \\
\text { me. }\end{array}$ & $\begin{array}{l}\text { Journal of } \\
\text { Advanced } \\
\text { Nursing }\end{array}$ & China & $\begin{array}{l}\text { Oitenta e cinco pacientes } \\
\text { participaram do estudo em } \\
2005,43 \text { no grupo estudo e } \\
42 \text { no grupo controle. Os } \\
\text { pacientes do grupo } \\
\text { controle receberam alta } \\
\text { hospitalar de rotina. Os } \\
\text { pacientes do grupo de } \\
\text { estudo receberam um } \\
\text { programa educacional } \\
\text { abrangente antes da alta e } \\
\text { um acompanhamento por } \\
\text { telefone padronizado de } 6 \\
\text { semanas iniciado pela } \\
\text { enfermeira. A qualidade de } \\
\text { vida da doença renal foi } \\
\text { medida para cada paciente } \\
\text { em três intervalos de } \\
\text { tempo: antes da } \\
\text { intervenção, na conclusão } \\
\text { da intervenção de } 6 \\
\text { semanas e } 6 \text { semanas após o } \\
\text { término do programa. }\end{array}$ & $\begin{array}{l}\text { Nesse estudo, foi } \\
\text { realizada análise de } \\
\text { variância de medidas } \\
\text { repetidas, modelo } \\
\text { linear geral. Efeitos } \\
\text { estatisticamente } \\
\text { significativos dentro } \\
\text { do grupo foram } \\
\text { encontrados para } \\
\text { sintomas } \\
\text { problemas, efeitos de } \\
\text { doença renal, sono, } \\
\text { função física, dor, } \\
\text { bem-estar emocional } \\
\text { e função social. Dessa } \\
\text { forma, foi descoberto } \\
\text { que o programa de } \\
\text { gerenciamento de } \\
\text { casos liderado por } \\
\text { enfermeiras pode ser } \\
\text { aplicado de forma } \\
\text { eficaz a pacientes em } \\
\text { diálise peritoneal. O } \\
\text { novo modelo de } \\
\text { atendimento é } \\
\text { particularmente útil } \\
\text { para melhorar o } \\
\text { bem-estar dos } \\
\text { pacientes na na } \\
\text { transição do hospital } \\
\text { para casa. }\end{array}$ \\
\hline
\end{tabular}

Fonte: Autoria Própria.

\section{DISCUSSÃO}




\section{A DP como escolha do paciente por modalidade de tratamento}

Sabe-se que a insuficiência Renal Crônica tende a levar o paciente a tomada de uma decisão difícil, onde ele deverá escolher o melhor método de tratamento possível para o seu cotidiano de vida. Para isso, o paciente deverá escolher as modalidades de diálise oferecidas para dar início ao processo de Terapia Renal Substitutiva (TRS) (ABUD et al., 2017; ZEE et al., 2018).

Durante a consulta, o paciente tem o direito e o dever de escolher a modalidade de tratamento adequada. Podendo ser a hemodiálise, Diálise Peritoneal Ambulatória ou Diálise Peritoneal Automatizada (DPA). Para isso, é necessário que o enfermeiro auxilie o paciente na sua tomada de decisão, esclarecendo e explicando as principais vantagens e desvantagens de cada uma das modalidades (SILVA et al., 2016; CAMPOS et al., 2019).

A escolha da diálise peritoneal como modalidade de tratamento deve ser pensada, pois é algo para o resto da vida ou até a ocorrência de um transplante renal. Logo, existem diversos fatores que auxiliam nessa tomada de decisão, no qual vai depender de como o indivíduo quer dar continuidade a sua vida realizado o processo de TRS.

De acordo com a literatura, a diálise peritoneal é considerada bastante segura e eficaz no desenvolvimento de TRS, trazendo alívio e seguridade para o doente renal crônico. Esse procedimento é considerado a forma menos agressiva e é realizado através de uma cirurgia com a implantação de um cateter na cavidade peritoneal da pessoa e ao longo do tratamento a próprio paciente irá realizar a troca das bolsas em seu ambiente domiciliar. No entanto, para que o tratamento seja realizado de forma adequada, o paciente deve adotar um estilo de vida diferenciado em relação a alimentação, medicamentos, higiene e cuidados com a saúde. Sendo assim, é notório que quem realiza a DP tem uma privacidade e independência maior do que indivíduos que fazem a hemodiálise, trazendo uma melhor qualidade de vida e bem-estar para o paciente (CHIANG et al., 2016; ABUD et al., 2017; CAMPOS et al., 2019).

Orientações necessárias que o enfermeiro deve fornecer para os pacientes em DP 
A DP é um exemplo de Terapia Substitutiva Renal que obriga o paciente a adotar um estilo de vida diferenciado, cercado de orientações e cuidados proporcionados principalmente pelo enfermeiro. Esse profissional da saúde é representado como um importante facilitador para esse paciente, pois ele irá orientar sobre o estado de saúde, exames, medicamentos, dieta entre outro, para que assim melhore sua qualidade de vida (CAMPOS et al., 2019).

Ao longo do tratamento por DP o enfermeiro juntamente com a equipe multifuncional fornece inúmeras orientações para seus pacientes, uma delas é quanto a alimentação, cuja deve ser suplementada por alimentos ricos em ácido fólico e cianocobalamina (vitamina B12), pois esses alimentos ajudam na maturação das hemácias, que por sua vez diminuem a anemia presente nos pacientes decorrentes da própria condição clínica. Ademais, devem fazer o consumo de fibras solúveis como gomas, mucilagens, entre outras, que são essências para a normalização do trânsito intestinal (SILVA et al., 2016; CAMPOS et al., 2019).

Outra orientação presente nos estudos é a importância de fazer atividades físicas, podendo ser exercícios de resistência e até mesmo atividades do cotidiano, já que proporcionam uma melhora na força e minimiza os sintomas da fadiga. Nesse sentido os exercícios físicos se mostram como um benefício para os pacientes de DP (SILVA et al., 2016; CAMPOS et al., 2019).

Os estudos também mostraram que as informações fornecidas pelo enfermeiro voltadas a prevenção e controle de infecções, são de suma importância, já que foi evidenciado que a sepse é considerada a segunda causa de morte em pacientes em diálise peritoneal, principalmente naqueles que apresentam mais de um episódio durante o tratamento. Já no Brasil, a maioria dos pacientes apresentaram infecção na saída do cateter e pericardite, que foram observados em um estudo realizado com 90 pacientes, na qual 42,2\% já tiveram peritonite, sendo que 15,5\% deles apresentaram dois ou mais episódios. Nesse sentido, destacamos a infecção como sendo a principal complicação da diálise peritoneal, seja na modalidade manual ou automática (CAMPOS et al., 2019).

Na orientação dos pacientes para higienização das mãos em domicílio é recomendado o uso de sabão glicerinado durante três minutos, ou álcool 70\% que é considerado mais eficaz, depois é retirado todos os adornos como pulseiras, anéis, relógios e, caso o paciente esteja com roupas de mangas compridas, é recomendado que as dobre até a altura dos cotovelos, 
antes da lavagem. Após a lavagem, é importância secar as mãos para minimizar os níveis de contaminação microbiana, dessa forma é utilizado papel toalha descartável ou toalha limpa. Além disso, é importante higienizar o orifício onde está inserido o cateter, com álcool 70\% e realizar a trocar o curativo diariamente, para minimizar riscos de infecções (CAMPOS et al.,2019).

Outro fator que contribui muito para a diminuição das complicações é o local que vai acontecer o manuseio e realização da DP domiciliar, o enfermeiro tem o papel de guiar a família ou os cuidadores a construção ou adaptação dos espaços que será realizado esse tratamento. $\mathrm{O}$ ambiente deve estar adaptado, higienizado e propício para o paciente realizar essa prática sem a ajuda dos profissionais da saúde. Abud (2017), em sua pesquisa, avaliou a estrutura física dos domicílios dos pacientes que realizam DP em domicilio. Assim, descobriu que a integridade do piso, o revestimento do teto, água tratada, rede de esgotamento sanitário e uma pia para a higienização das mãos próxima onde irá ser realizado o procedimento da diálise irá diminuir as chances de infecções, visto que, facilita a limpeza do local, reduz o risco de contaminação do material e diminui a exposição dos pacientes a riscos desnecessários (ABUD et al., 2017).

Com relação a higienização do local, é essencial uma limpeza diariamente, objetivando a diminuição da possibilidade de infecção. Outrossim, manter o material que será utilizado na DP sempre higienizado, utilizando álcool 70, além de armazenar em locais que não sejam ensolarados, úmido ou em contato direto com o chão. Destaca-se também a importância das orientações sobre o sono principalmente aos idosos em DP, no qual irá ajudar a reduzir os acidentes, doenças relacionadas ao sono e a qualidade de vida desses pacientes. No entanto, se houver casos de insônia o profissional da saúde deve auxiliar no aconselhamento e tratamento (CHOW; WONG, 2010).

\section{Principais diagnósticos de enfermagem e intervenções que o enfermeiro pode realizar em pacientes com DP}

A DP é frequentemente considerada um método fácil e eficiente de terapia renal substitutiva, que proporciona uma melhor qualidade de vida aos pacientes. No entanto, 
alguns problemas podem ocorrer por falta de orientações e manejos clínicos que ocasionam o declínio da função renal residual (ABUD et al., 2017).

Inicialmente o risco de infecção que segundo os estudos, está presente em todos os pacientes em DP. Essa complicação é explicada devido os pacientes serem expostos a procedimentos invasivos e apresentarem uma doença crônica. Esse problema é a segunda causa de morte, sendo de aproximadamente 18\% devido à peritonite (SILVA et al., 2016; CAMPOS et al., 2019).

A peritonite está possivelmente relacionada a presença de infecção no túnel ou no sítio de saída do cateter peritoneal. Esse fator leva a falência do peritônio, proporcionando transtornos e uma possível causa de transferência dos pacientes de dialise peritoneal para hemodiálise (CAMPOS et al., 2019). Essa complicação é a principal causa de hospitalização e falhas clinicas (YANG et al., 2012; CHEN et al., 2019). Mesmo com o treinamento proporcionado pela equipe de saúde sobre o tratamento, métodos assépticos, materiais utilizados e cuidados no domicilio, os pacientes ainda apresentavam dificuldades e duvidas no manuseio do cateter da DP. Desse modo, é importante focalizar ainda mais no ensino dos procedimentos ao paciente e em minimizar a infecção no peritônio que em sua maioria são situadas na saída do cateter e, em alguns casos, possivelmente adquirida por erros de procedimento durante $\mathrm{o}$ autocuidado com o cateter de diálise peritoneal.

A fadiga também foi um diagnóstico presente nos pacientes com DP. Esse quadro é ocasionado devido a deficiência na difusão do oxigênio, levando as células a produzirem uma grande quantidade de ácido láctico, e assim, provocando uma perda progressiva da estrutura muscular (SILVA et al., 2016; CAMPOS et al., 2019). Esse estado afeta diretamente a vida diária do paciente, deixando o paciente mais apto a ficar em seus leitos, minimizando assim sua funcionalidade (SILVA et al., 2016). Nesse caso uma equipe multiprofissional, não só a enfermagem, pode intervir nesse diagnóstico conservando a energia do paciente e reavaliando a sua terapia nutricional caso haja a necessidade de mudar a sua dieta.

A Deambulação Prejudicada também esteve presente no estudo e pode ser conceituado como uma limitação dos movimentos físicos independentes. Esse problema contribui para um estilo de vida sedentário, o que irá comprometer a saúde cardiovascular além de induzir à perda de proteínas musculares, proporcionando a atrofia muscular, já que o paciente se encontrará com falta de condicionamento e inativo das atividades físicas (SILVA 
et al., 2016; CAMPOS et al., 2019). O enfermeiro deverá auxiliar o cliente na efetuação de exercícios que promovam a restauração das funções anatômicas e voluntárias do corpo, além de aumentar a resistência muscular.

Temos também a constipação que é definida como a dificuldade e/ou ausência de massa fecal por um determinado período e é apresentado pelos pacientes em DP. A causa desse problema provém principalmente da baixa ingesta hídrica; do uso de certos medicamentos, como a sinvastatina e o omeprazol; a idade avançada e o sedentarismo. (SILVA et al., 2016).

Outro fenômeno presente nos pacientes com DP é a dor aguda, que é identificada como uma experiência sensorial e emocional desagradável que está relacionada a limitações pessoais e sociais decorrentes da doença e do tratamento (SILVA et al., 2016; ABUD et al., 2017). Nessa situação o médico pode auxiliar realizando a prescrição de analgésicos-antipiréticos ou anestésicos locais para supressão da dor e o enfermeiro irá realizar a administração.

\section{A assistência prestada pelo enfermeiro ao paciente em processo de DP}

A assistência que o enfermeiro presta ao paciente que está em processo de DP é de suma importância, pois é ele que contribuí para a redução de complicações que possam interferir no tratamento dialítico, proporcionando ao paciente uma maior seguridade e confiança na qualidade do serviço ofertado pela unidade de Diálise Peritoneal (CAMPOS et al., 2019).

A equipe de enfermagem deve estar sempre disponível ao paciente com doença renal crônica, pois são exigidos cuidados desde o pré-operatório com circunspeção e manuseio clínicos até a implantação do cateter no paciente, no qual o enfermeiro sempre deve estar atento a possíveis complicações pós-cirúrgicas, como o aspecto do curativo no local da implantação do cateter, extravasamento de líquidos, edema e queixas do paciente (ABUD et al., 2017; CAMPOS et al., 2019).

Outro papel importante para o enfermeiro é estar presente não apenas prestando cuidados técnicos no processo dialítico, mas também o acolher no momento da descoberta da doença com um apoio psicológico e afetivo. Ele precisa incentivar o paciente a seguir o método dialítico da forma adequada, proporcionado conforto, para que o indivíduo se sinta 
seguro para expressar seus medos e consequentemente esclarecer qualquer dúvida sobre o tratamento (IMADA 2012; CAMPOS et al., 2019).

Dessa forma, o objetivo da enfermagem na Diálise Peritoneal é apoiar o paciente para que a terapia dialítica possa ser realizada de maneira correta e segura como parte do estilo de vida daquele paciente, para que assim possa detectar rapidamente anormalidades fisiológicas e identificar possíveis complicações que se possa ter ao longo do tratamento (IMADA, 2012; CAMPOS et al., 2019). Além disso, o enfermeiro também avalia e dá suporte ao paciente, por meio de treinamentos teóricos, motivações e habilidades manuais, que são direcionadas as especificidades de cada enfermo, para que assim, ele possa colaborar em seu tratamento (SILVA et al., 2016).

De acordo com os estudos, nota-se que a experiência do enfermeiro facilita e traz uma maior eficácia ao tratamento dialítico. Isso se deve ao fato de que essa vivência vai trazer uma maior segurança ao enfermeiro e consequentemente uma maior cautela aos cuidados necessários durante o tratamento, como avaliar os exames laboratoriais frequentemente, analisar as manifestações corporais do paciente, observar possíveis infecções através de sinais e sintomas e principalmente higienizar as mãos da forma correta antes e depois de cada procedimento. Assim trazendo uma maior proteção ao paciente (YANG et al., 2012; CAMPOS et al., 2019).

Ademais, vale ressaltar que os cuidados básicos são essenciais para diminuir a chance de infecções. Dessa forma, a equipe de enfermagem deve auxiliar seus clientes em relação ao autocuidado e aprimoramento da sua autoeficácia através de treinamentos realizados continuamente para que assim, evitem dificuldades em atender esses indivíduos (IMADA, 2012).

Segundo os estudos, a Sociedade Internacional de Diálise Peritoneal (ISPD), afirma que problemas com a lavagem das mãos e o uso de máscaras, além da Diabetes Mellitus são as principais causas de peritonite. Além disso, fatores sociodemográficos, nutricionais, circunstâncias climáticas, modalidade de DP são descritos como possíveis fatores de risco associados ao desenvolvimento de peritonite. Logo, é necessário que o processo de preparação para a realização da DP seja feito utilizando técnicas assépticas para a lavagem das mãos e obrigatoriamente o uso de uma máscara cirúrgica em conjunto com uma máscara protetora facial, promovendo o controle da disseminação de microrganismos expelidos pelo 
nariz ou pela boca, agindo como bloqueio mecânico, além de melhorar a assistência fornecida pelo enfermeiro (CHIANG et al., 2016; KARADAG, 2019; CHEN, 2019).

Por fim, podemos destacar que a sistematização da assistência de enfermagem (SAE) seria algo essencial para que o enfermeiro possa identificar com mais facilidade as complicações geradas pelos métodos dialíticos, garantindo uma solução mais ágil e eficiente. A SAE utiliza o processo de enfermagem que envolve cinco momentos: investigação ou histórico de enfermagem, diagnóstico de enfermagem, planejamento, implementação e avaliação. Essas etapas são utilizadas para organizar o cuidado, anotar suas práticas e observar as necessidades humanas em relação ao estado de saúde de cada paciente, com o objetivo de identificar intervenções e futuros resultados (IMADA, 2012; SILVA et al., 2016; CAMPOS et al., 2019).

\section{CONCLUSÃO}

O indivíduo que adquiri insuficiência renal crônica terá inúmeras incapacidades ao longo da vida. Normalmente, essa pessoa deverá optar por um dos tratamentos que possa substituir a função renal do seu organismo. A Diálise Peritoneal acaba se tornando um método dialítico bastante eficaz que facilita a mudança do estilo de vida da pessoa.

O enfermeiro acaba tendo um papel fundamental em todo esse processo, ajudando na escolha da DP como modalidade de tratamento e na orientação para realizar esse procedimento em ambiente domiciliar. É ele quem esclarece e mostra as principais vantagens e desvantagens da diálise peritoneal. Além disso, o profissional de enfermagem irá orientar o paciente durante todo o tratamento, como a alimentação, exercícios que devem ser realizados, principais cuidados com o cateter e o seu orifício de saída para evitar possíveis infecções.

Outrossim, a equipe de enfermagem ainda deverá auxiliar o cliente a efetuar a dialise peritoneal em casa, ensinando todo o procedimento que deve ser feito e explicando como o ambiente deve está para realizar o método. O enfermeiro também ficará responsável por dar apoio psicológico e afetivo caso o paciente necessite. Dessa forma, percebe-se que a 
enfermagem é bastante essencial para o indivíduo que implementa a dialise peritoneal no seu cotidiano, facilitando o novo estilo de vida daquela pessoa.

\section{REFERÊNCIAS}

ABUD, Ana Cristina Freire; ZANETTI, Maria Lúcia; INAGAKI, Ana Dorcas de Melo; SANTOS, André Faro; LIMA, Raíssa Santana; MOURA, Renata Pinto. Ambiência domiciliar para a realização da diálise peritoneal. Revista Enfermagem UERJ, Rio de Janeiro, v. 25, ed. 15210, 2017. DOI https://doi.org/10.12957/reuerj.2017.15210. Disponível em: https://www.e-publicacoes.uerj.br/index.php/enfermagemuerj/article/view/15210/22381. Acesso em: 26 ago. 2021.

BRASIL. Diretrizes clínicas para o cuidado ao paciente com doença renal crônica-DRC no Sistema Único de Saúde. Secretaria de Atenção à Saúde, v. 1, p. 1-37, 2014.

BEZERRA, Cícero Itálo Leite. o paciente renal crônico e a escolha do método dialítico: uma análise da influência de ansiedade, estresse e depressão. 2017. Tese de Doutorado. Universidade de São Paulo.

CHIANG, Pei-Chun; HOU, Jia-Jeng;JONG, Ing-Ching; HUNG, Peir-Haur; HSIAO, Chih-Yen; MA , Tsung-Liang; HSU, YuehHan. Factors Associated with the Choice of Peritoneal Dialysis in Patients with End-Stage Renal Disease. BioMed Research International, [s. l.], v. 2016, 2016. DOI https://doi.org/10.1155/2016/5314719. Disponível em: https://www.hindawi.com/journals/bmri/2016/5314719/. Acesso em: 26 ago. 2021.

CHOW, Susan Ka Yee; WONG, Frances KY. Health-related quality of life in patients undergoing peritoneal dialysis: effects of a nurse-led case management programme. Journal of advanced nursing, v. 66, n. 8, p. 1780-1792, 2010. DOI https://doi.org/10.1111/j.1365-2648.2010.05324.x. Disponível em: https://pubmed.ncbi.nlm.nih.gov/20557392/. Acesso em: 26 ago. 2021

CAMPOS, Moiziara Xavier Bezerra; DUTRA, Eva Jordana de Oliveira; SILVA, Carlos Jordão de Assis; MENEZES, Harlon França de; SANTOS, Rebecca Stefany da Costa; DA SILVA, Richardson Augusto Rosendo. Pacientes em diálise peritoneal: associação entre diagnósticos de enfermagem e seus componentes. Acta Paulista de Enfermagem, [s. l.], v. 32, ed. 6, p. 651-8, 2019. DOI https://doi.org/10.1590/1982-0194201900090. Disponível em: https://www.scielo.br/j/ape/a/KVdYHdQjGX7ypNMqHSmB6Fq/?lang=pt\&format=html. Acesso em: 26 ago. 2021.

CHEN, Hsiao-Ling; TARNG, Der-Cherng; HUANG, Lian-Hua. Risk factors associated with outcomes of peritoneal dialysis in Taiwan. Medicine, [s. l.], v. 98, ed. 6, 2019. DOI https://doi.org/10.1097/MD.0000000000014385. Disponível em: https://www.ncbi.nlm.nih.gov/pmc/articles/PMC6380716/. Acesso em: 26 ago. 2021..

SOUSA, Luís Manuel Mota de; MARQUES-VIEIRA, Cristina Maria Alves; SEVERINO, Sandy Silva Pedro; ANTUNES, Ana Vanessa. A metodologia de revisão integrativa da literatura em enfermagem. Revista investigação em enfermagem, [s. l.], v. 17, p. 17-26, 2017.

MORAES, Alice da Silva; SOUZA, Airle Miranda de; SENA, Teresa Christina da Cruz Bezerra de; FALCÃO, Luiz Fábio Magno; CORREAA, Victor Augusto Cavaleiro. Alterações no desempenho ocupacional de pessoas com doença renal crônica em diálise peritoneal. Revista Família, Ciclos de Vida e Saúde no Contexto Social, [s. l.], p. 591-599, 2018. DOI https://doi.org/10.18554/refacs.v6i0.3129. Disponível em: http://seer.uftm.edu.br/revistaeletronica/index.php/refacs/article/view/3129. Acesso em: 26 ago. 2021.

DA SILVA, Claudenizio Nunes da; BARBOSA, Eliane Silva; DA SILVA, Elisonia Nunes; AOYAMA, Elisângela de Andrade; LIMA, Ronaldo Nunes. Atuação do enfermeiro no tratamento de diálise peritoneal ao portador de insuficiência renal crônica. Revista Brasileira Interdisciplinar de Saúde, [s. l.], v. 1, n. 3, p. 66-72, 2019.

IMADA, Akio. Care for patients carrying out dialysis therapy at home. In: Home Dialysis in Japan. Karger Publishers, 2012.p. $93-98$ 
KARADAG, Ezgi. The effect of a self-management program on hand-washing/mask-wearing behaviours and selfefficacy level in peritoneal dialysis patients: a pilot study. Journal of renal care, v. 45, n. 2, p. 93-101, 2019.

MENDES, Marcela Lara; ALVES, Camila Albuquerque; BUCUVIC, Edwa Maria; DIAS, Dayana Bitencourt; PONCE, Daniela. Iálise peritoneal como primeira opção de tratamento dialítico de início não planejado. Brazilian Journal of Nephrology, [s. l.], v. 39, n. 4, p. 441-446, 2017. DOI https://doi.org/10.5935/0101-2800.20170077. Disponível em: https://www.scielo.br/j/jbn/a/KBqwR6jC3JsG6ZVLtzcVhJM/abstract/?lang=pt\#. Acesso em: 26 ago. 2021.

MUSCAT, Priscilla; WEINMAN, John; FARRUGIA, Emanuel; CALLUS, Roberta; CHILCOT, Joseph. Illness perceptions predict distress in patients with chronic kidney disease. BMC Psychology, [s. l.], v. 9, n. 1, p. 1-13, 2021. DOI https://doi.org/10.1186/s40359-021-00572-z. Disponível

em: https://bmcpsychology.biomedcentral.com/articles/10.1186/s40359-021-00572-z. Acesso em: 26 ago. 2021.

NEVES, Precil Diego Miranda de Menezes; SESSO, Ricardo de Castro Cintra; THOMÉ, Fernando Saldanha; LUGON, Jocemir Ronaldo; NASCIMENTO, Marcelo Mazza. Censo Brasileiro de Diálise: análise de dados da década 20092018. Brazilian Journal of Nephrology, [s. l.], v. 42, n. 2, p. 191-200, 2020. DOI https://doi.org/10.1590/2175-8239-JBN2019-0234. Disponível em: https://www.scielo.br/j/jbn/a/Dbk8Rk5kFYCSZGJv3FPpxWC/?lang=pt\&format=html. Acesso em: 26 ago. 2021.

SILVA, Richardson Augusto Rosendo da; BEZERRA, Moiziara Xavier; NETO, Vinicius Lino de Souza; MENDONÇA, Ana Elza Oliveira de; SALVETTI, Marina de Góes. Diagnósticos, resultados e intervenções de enfermagem para pacientes em diálise peritoneal. Acta Paulista de Enfermagem, [s. l.], v. 29, n. 5, p. 486-493, 2016. DOI https://doi.org/10.1590/1982-0194201600069. Disponível

https://www.scielo.br/j/ape/a/3hvJKqPxG5tvhdhKrhsBPNB/?format=html\&lang=pt. Acesso em: 26 ago. 2021.

ANG, Zhikai; XU, Rong; ZHUO, Min; DONG, Jie. Advanced nursing experience is beneficial for lowering the peritonitis rate in patients on peritoneal dialysis. Peritoneal Dialysis International, [s. l.], v. 32, n. 1, p. 60-66, 2012. DOI https://doi.org/10.3747/pdi.2010.00208. Disponível https://journals.sagepub.com/doi/abs/10.3747/pdi.2010.00208. Acesso em: 26 ago. 2021.

ZEE, Jarcy; ZHAO, Junhui; SUBRAMANIAN, Lalita; PERRY, Erica; BRYANT, Nicole; MCCALL, Margie; RESTOVIC, Yanko; TORRES, Delma; ROBINSON, Bruce; PISONI, Ronald; TENTORI, Francesca. Perceptions about the dialysis modality decision process among peritoneal dialysis and in-center hemodialysis patients. BMC nephrology, [s. l.], v. 19, n. 1, p. 1-10, 2018. DOI https://doi.org/10.1186/s12882-018-1096-x. Disponível em: https://link.springer.com/article/10.1186/s12882-018-1096-x. Acesso em: 26 ago. 2021.

(cc) Br

Este trabalho está licenciado com uma Licença Creative Commons - Atribuição 4.0 Internacional. 